\title{
Modelado del Proceso de Fritura del Ñame (Dioscorea alata) mediante Mediciones Reológicas usando la Metodología de Superficie de Respuesta
}

Armando Alvis ${ }^{*^{1}}$ y Carlos A. Vélez ${ }^{2}$

(1) Universidad de Córdoba, Facultad de Ciencias Agrícolas, Departamento de Ingeniería de Alimentos, Carrera 6 No 76-103, Km 3, vía Cereté, Córdoba-Colombia (e-mail: aalvis@hotmail.com)

(2) Universidad del Valle, Facultad de Ingeniería, Escuela de Ingeniería de Alimentos,

Apdo. Aéreo 25360, Cali-Colombia

*autor a quien debe ser dirigida la correspondencia

\section{Resumen}

En este trabajo se llevó a cabo un estudio acerca de los efectos que producen sobre el freído de trozos de ñame (Dioscorea alata) el tiempo de freído (1-9 minutos) y la temperatura del aceite (140$180^{\circ} \mathrm{C}$ ). Se analizan las interacciones de estas variables con algunas propiedades de textura tales como fuerza de fractura en flexión y fuerza máxima de penetración. El análisis se realizó mediante gráficos de superficies de respuesta para cada una de las variables evaluadas y se obtuvieron modelos matemáticos para establecer la relación entre estas variables. Se concluye que la textura está mayormente influenciada por el tiempo de freído.

Palabras clave: freído de ñame, Dioscorea alata, propiedades reológicas, superficie de respuesta

\section{Modeling the Frying Process of Yam (Dioscorea alata) through Rheological Measurements using Response Surface Methodology}

\begin{abstract}
The effects of frying time (1-9 min.) and oil temperature $\left(140-180^{\circ} \mathrm{C}\right)$ on deep-fat frying of yam chips (Dioscorea alata) were studied in this work. Interactions of these variables with textural properties such as fracture stress in flexion and maximum penetrating force. Surface response graphs were used to analyze the influence of each variable and mathematical models were obtained to establish the relation among these variables. It was concluded that texture is mainly influenced by frying time.
\end{abstract}

Keywords: frying yam, Dioscorea alata, rheological properties, surface response method 


\section{INTRODUCCIÓN}

El ñame (Dioscorea alata), debido a la cantidad de carbohidratos que proporciona, es un alimento básico para muchos pobladores de las regiones tropicales y subtropicales húmedas del occidente de África, Asia y el Caribe (Coursey y Haynes, 1970; Bradbury y Holloway, 1987; ONU, 1990; FAO/FAOSTAT, 2002); en esta última región, en la Costa Atlántica Colombiana (zona de mayor producción de ñame), se cultivan alrededor de 29.757 ha, que involucran a 9.000 familias de pequeños agricultores, con un área promedio de 1,4 ha por familia, un rendimiento promedio de 11,9 Ton/ha y una producción de 264.500 Ton/año (Minagricultura, 2003).

El sistema de comercialización de los pequeños productores se caracteriza por bajos volúmenes, escasa infraestructura de acopio, transporte, almacenamiento y transformación y un alto grado de intermediación que lo hace complejo por falta de organización de los productores (Bradbury y Holloway, 1987; ONU, 1990). Además, la estacionalidad de la producción, hace que exista una sobreoferta en determinada época del año, lo que se refleja en la reducción de precios, caída de la demanda, pérdidas poscosecha del orden del 30 a $40 \%$. En Colombia, donde el $78 \%$ de la producción se dirige al mercado en fresco, no se conocen transformaciones tecnológicas (Rodríguez, 1996), mientras que en África sólo es tradicional la preparación de harina y en los últimos años se comenzó a producir trozos secos.

La transformación de los productos ricos en almidón mediante el freído se han realizado principalmente para papa y yuca, sin que se conozca publicaciones hasta la fecha acerca del comportamiento del proceso de freído del ñame. El freído es una operación de transferencia simultánea de masa y calor, donde el cocimiento en aceite imparte varios atributos de calidad deseables como el sabor, textura, color, olor a los productos alimenticios. Estos cambios deseables dados a los alimentos cocinados son un efecto combinado de formación de costra, pérdida de humedad, ganancia de aceite, desnaturalización de las proteínas, gelatinización de los almidones y cuantiosos cambios microestructurales en el interior del alimento (Blumenthal, 1991; Sagui y Pinthus, 1995; Sagui et al., 1998; Aguilera, 1997).

La textura de los alimentos es una de las cualidades primarias que determinan su calidad sensorial. Su definición no es fácil porque es el resultado de la acción de estímulos de diversa naturaleza. Se ha definido como "conjunto de propiedades reológicas y de estructura (geométricas y de superficie) de un producto perceptibles por los mecano-receptores, los receptores táctiles y en ciertos casos, por los visuales y los auditivos" (Costell et al., 1997; Pedreschi y Moyano, 2005).

En papas fritas la textura es el principal factor que determina la aceptabilidad y depende tanto de la materia prima como del procesamiento y período de posfreído. Una papa frita de buena calidad es aquella que tiene una corteza exterior crocante pero no dura ni "chiclosa" y un centro húmedo, como papa cocida. La característica crocante de la corteza proviene probablemente de la condición vítrea del almidón a baja humedad $(\sim 2 \%)$ la que se pierde al migrar el agua desde el centro húmedo (Aguilera, 1997).

Taiwo y Baik (2007), estudiaron los efectos de diversos pretratamientos (escaldado, congelación y deshidratación y un producto sin tratar) sobre la reducción de las propiedades texturales en batatas fritas a $170{ }^{\circ} \mathrm{C}$ durante un tiempo de 0.5 a 5 minutos y observaron que estos pretratamientos mejora las propiedades texturales (dureza, adhesividad y cohesión) de las muestras de productos fritos después de $120 \mathrm{~s}$. Ikoko y Kuri (2007), estudiaron los cambios en la composición durante la fritura y deshidratación osmótica de tajadas de plátano y encontraron que el producto crudo posee un alto grado de dureza al realizar la prueba de flexión y un bajo grado de adhesividad cuando se expuso a la deshidratación osmótica, mientras que, el producto freído alcanza el mayor grado de dureza a los tiempos de 5 a 10 minutos, mientras que a 2 minutos alcanza los niveles más bajos.

Teniendo en cuenta que la textura de los productos fritos representa un atributo de calidad de extrema importancia, el objetivo del presente estudio es determinar la influencia de los parámetros del proceso de freído (temperatura del aceite y tiempo de freído) sobre las características texturales del ñame, mediante análisis de superficie de respuesta. 


\section{MATERIALES Y MÉTODOS}

Para el estudio se utilizó la variedad Pico de Botella que se cultiva en la región norte de Colombia. Los ñames fueron pelados, lavados y cortados en forma de paralelepípedo de dimensiones $0.8 \times 0.8$ $\times 4 \mathrm{~cm}$.

El proceso de freído se llevó a cabo en una freidora de acero inoxidable de 5 litros de capacidad con control automático de temperatura Marca MKE (Indianápolis - EUA) y se utilizó aceite de soya refinado. Una vez alcanzadas las temperaturas de freído deseadas, se sumergieron simultáneamente 30 trozos de ñame y para cada tiempo de freído establecido el producto se extrajo del baño y se dejó escurrir sobre toallas de papel absorbente para eliminar la grasa superficial.

Se empleó un diseño estadístico de experimentos factorial hexagonal (Gacula y Singh, 1984; Montgomery, 2002), con 5 niveles de tiempo de freído (1, 3, 5, 7 y 9 min.) y tres temperaturas del aceite $\left(140,160\right.$ y $\left.180^{\circ} \mathrm{C}\right)$. Los limites inferiores y superiores y el punto central del diseño fueron codificados como $-1,+1$ y 0 respectivamente. La matriz consta de 18 experimentos, tres de los cuales son réplicas del punto central $\left(160{ }^{\circ} \mathrm{C}\right.$ cada $5 \mathrm{~min}$.) y se desarrollaron siguiendo el orden aleatorio indicado (Tabla 1).

La metodología de superficie de respuesta fue aplicada a las variables respuesta usando el software estadístico comercial Desing Expert Versión 5.0 (Stat-Ease, Minneapolis, EUA). Fueron ajustados polinomios de segundo orden a los datos para obtener ecuaciones de regresión para las variables de respuesta analizadas. El gráfico de las superficies de respuesta, el análisis de varianza y los coeficientes de determinación $\left(R^{2}\right)$ fueron generados con el mismo software.

Tabla 1: Matriz del diseño de experimentos

\begin{tabular}{|c|c|c|}
\hline Experimento & Tiempo $(\mathrm{t})$ & Temperatura $(\mathrm{T})$ \\
\hline 1 & 0 & -1 \\
\hline 2 & 0.5 & -1 \\
\hline 3 & 0 & 0 \\
\hline 4 & 0 & 0 \\
\hline 5 & 0 & 0 \\
\hline 6 & -1 & 0 \\
\hline 7 & 0.5 & 0 \\
\hline 8 & -0.5 & -1 \\
\hline 9 & -1 & 1 \\
\hline 10 & -0.5 & 0 \\
\hline 11 & 1 & 0 \\
\hline 12 & 0 & 0 \\
\hline 13 & 0.5 & 1 \\
\hline 14 & 1 & 1 \\
\hline 15 & -1 & -1 \\
\hline 16 & 1 & -1 \\
\hline 17 & -0.5 & 1 \\
\hline 18 & 0 & 1 \\
\hline
\end{tabular}

Se realizaron pruebas de flexión y penetración para el análisis de textura en una máquina Instron ${ }^{\circledR}$ modelo 1140. Para los ensayos de flexión se utilizó un puente de flexión de tres puntos a una distancia de $2 \mathrm{~cm}$., flexionando la muestra en el centro hasta fractura, de cuya curva se obtuvo la fuerza de fractura $\left(F_{f}\right)$.

Para la prueba de penetración se utilizó un vástago cilíndrico de $\Phi=4,6 \mathrm{~mm}$ para obtener la fuerza máxima de penetración $\left(F_{p}\right)$ de la curva fuerza vs distancia, la deformación $\left(D_{p}\right)$ expresada en metros, como la distancia correspondiente al valor de $F_{f}$ y la pendiente como la relación Información Tecnológica Vol. - 19 No5 - 2008 
$F_{p} / D_{p}=E_{a p}$ expresada en $\mathrm{N} / \mathrm{m}$ como medida empírica del módulo elástico. En todos los casos anteriores, las pruebas se hicieron con 5 réplicas, a la velocidad de deformación de $5 \mathrm{~cm} / \mathrm{min}$ y a la temperatura ambiente $\left(25^{\circ} \mathrm{C}\right)$.

\section{RESULTADOS Y DISCUSION}

En la tabla 2 se muestran los coeficientes de las ecuaciones de regresión ajustadas para la fuerza de fractura, fuerza máxima de penetración, deformación y pendiente, utilizados para establecer la influencia de la temperatura y el tiempo de freído sobre las características texturales del ñame. Como puede observarse, el tiempo de freído es altamente significativo para la fuerza de fractura, deformación y pendiente $(p \leq 0.01)$ y menos significativo para la fuerza máxima de penetración $(p \leq 0.05)$. El coeficiente cuadrático para el tiempo de freído es altamente significativo para la fuerza de fractura y Fuerza máxima de penetración $(p \leq 0.01)$ mientras que para la es menos significativo $(p \leq 0.05$ ). Por otra parte, puede observarse que la temperatura de freído no es significativa para la fuerza de fractura, mientras que altamente significativa para la fuerza máxima de penetración y la deformación ( $p \leq 0.01$ ) y menos significativa para la pendiente ( $p \leq 0.05$ ). El coeficiente cuadrático de la temperatura no es significativo para la fuerza de fractura y altamente significativo para la pendiente $(p \leq 0.01)$ y menos significativo para la fuerza máxima de penetración $(p \leq 0.05)$, la interacción de ambos parámetros resultó altamente significativa para la fuerza de fractura, fuerza máxima de penetración y pendiente $(p \leq 0.01)$.

Tabla 2: Información estadística para las variables de respuesta Fuerza de fractura, fuerza máxima de penetración, deformación y pendiente (t: Tiempo de freído (min.), T: Temperatura $\left({ }^{\circ} \mathrm{C}\right),{ }^{*} \alpha=5 \%$, ${ }^{* *} \alpha=1 \%$, NS: No significativo, $R^{2}=$ coeficiente de determinación)

\begin{tabular}{|c|c|c|c|c|}
\hline & Fuerza de fractura & $\begin{array}{c}\text { Fuerza máxima de } \\
\text { penetración }\end{array}$ & Deformación & Pendiente \\
\hline Constante & 8.56 & 22.7 & $3.98 \times 10^{-3}$ & 6634.34 \\
\hline $\mathrm{t}$ & $1.87^{* *}$ & $-1.0^{*}$ & $7.60 \times 10^{-4^{* *}}$ & $-1206.67^{* *}$ \\
\hline $\mathrm{T}$ & $0.21 \mathrm{NS}$ & $1.7^{\star *}$ & $2.90 \times 10^{-4^{* *}}$ & $276.67^{*}$ \\
\hline $\mathrm{t}^{2}$ & $5.06^{* *}$ & $2.2^{\star *}$ & - & $676.77^{*}$ \\
\hline $\mathrm{T}^{2}$ & $-1.34 \mathrm{NS}$ & $1.3^{*}$ & - & $-809.39^{\star *}$ \\
\hline $\mathrm{tT}$ & $-2.72^{\star *}$ & $-1.6^{* *}$ & - & $-730.00^{* *}$ \\
\hline $\mathrm{R}^{2}$ & 0.87 & 0.86 & 0.81 & 0.87 \\
\hline
\end{tabular}

Las ecuaciones de regresión ajustadas se utilizaron para obtener los gráficos de las superficies de respuesta y visualizar los efectos del tiempo y la temperatura sobre las variables analizadas.

$F_{f}=8.56+1.87 t+5.06 t^{2}-2,72 t T$

En la figura 1 se aprecia que a tiempos intermedios (3 - 5 min.) se obtienen los valores mínimos de la fuerza de fractura en flexión $\left(F_{f}\right)$ y prácticamente no existe mayor influencia de la temperatura, esto se debe al ablandamiento de los tejidos durante los primeros minutos de fritura por la gelatinización de los almidones que hacen el producto más blando (Anderson et al., 1994), mientras que el desarrollo de la corteza se da a medida que pasa el tiempo de fritura, en el producto se refleja la ruptura de la capa exterior del trozo de ñame cuando la flexión se da hasta fractura. Un comportamiento similar encontró Pedreschi y Moyano (2005) en el freído de rodajas de papa. A tiempos superiores (4 o 5 minutos) se presenta con mayor intensidad el desarrollo de la corteza; ésta no permite que el agua se desplace desde el interior del producto hasta la superficie quedando atrapada en el interior del mismo ocasionando fragilidad, el endurecimiento de la corteza se da a tiempos mayores de 7 minutos obteniéndose los mayores valores de fuerza de fractura. 


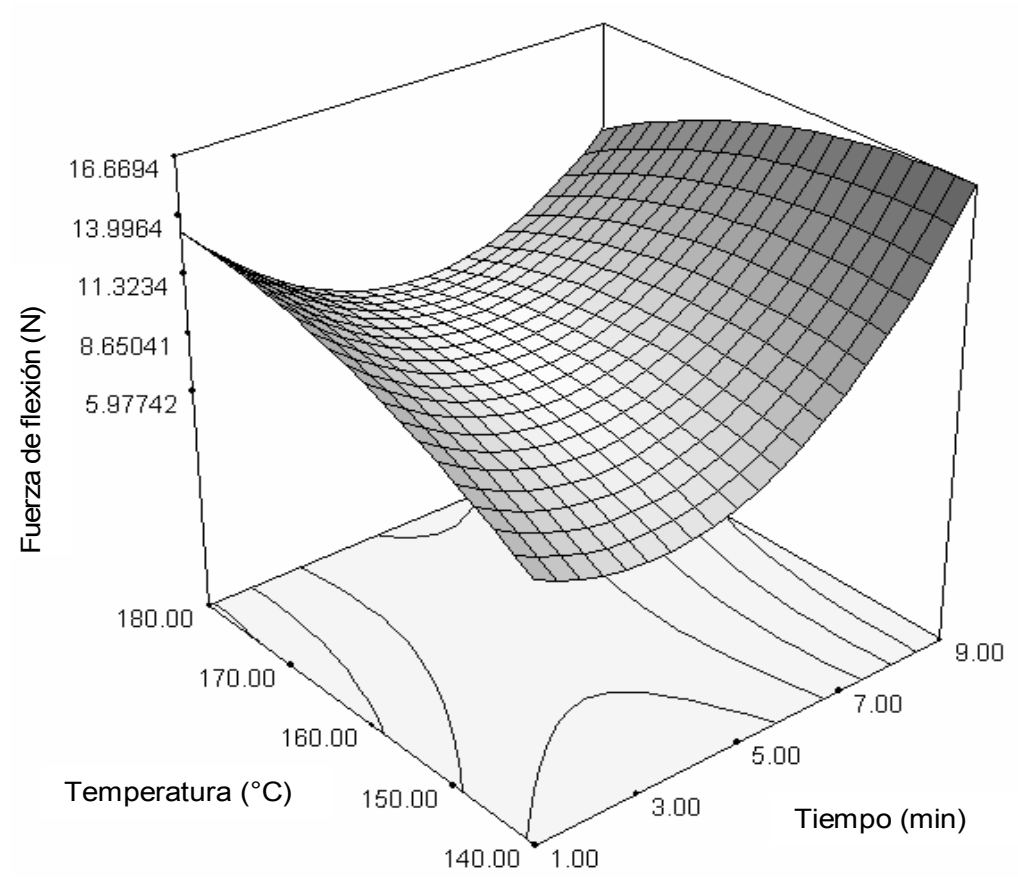

Fig. 1: Gráfico de superficie de respuesta para la fuerza de fractura

En los resultados de los coeficientes del modelo (1) se aprecia que el tiempo de freído ofrece la influencia más significativa, mientras que la temperatura del aceite por sí sola, no ofrece efecto significativo.

Los resultados de la prueba de penetración para la variable Fuerza Máxima de Penetración, muestran que la influencia del tiempo de freído y la temperatura del aceite se pueden explicar mediante el siguiente modelo cuadrático:

$F_{p}=22.7-1.0 t+1.7 T+2.2 t^{2}+1.3 T^{2}-1.6 t T$

El modelo cuadrático (2), permite apreciar cómo influye la temperatura y el tiempo de freído en la fuerza máxima de penetración y los dos factores logran la mayor influencia a temperaturas y tiempos de freído mayores, aunque ambos influyen sobre la fuerza máxima de penetración, la temperatura tiene mayor influencia. En la figura 2 se muestra el gráfico de superficie de respuesta correspondiente.

Se aprecia nuevamente como a los tiempos de fritura intermedios se alcanzan los valores más bajos de $\left(F_{p}\right)$ y a tiempos mayores del proceso los más elevados. Estas observaciones son semejantes a las analizadas para la fuerza de fractura; pero los valores más altos de la fuerza máxima de fractura se dan a mayor temperatura. Esto concuerda con los resultados obtenidos por Pedreschi y Moyano (2005) en cuanto a la afectación de la textura durante la fritura de rebanadas de papa evaluada a través de una prueba de penetración, por lo que en este sentido ambos tubérculos parecen comportarse de forma semejante; resultados similares encontraron Taiwo y Baik (2007).

Para el caso de la deformación $\left(D_{p}\right)$ del paralelepípedo frito calculada mediante la prueba de penetración, se obtuvo el modelo lineal siguiente:

$D_{p}=3.98 \times 10^{-3}+7.6 \times 10^{-4} t+2.9 \times 10^{-4} T$

Del modelo de la ecuación 3, se aprecia que el tiempo de freído ofrece un efecto superior en relación con el provocado por la temperatura. En la figura 3 se muestra el gráfico de la superficie de respuesta descrita por este modelo.

Información Tecnológica Vol. - 19 No5 - 2008 


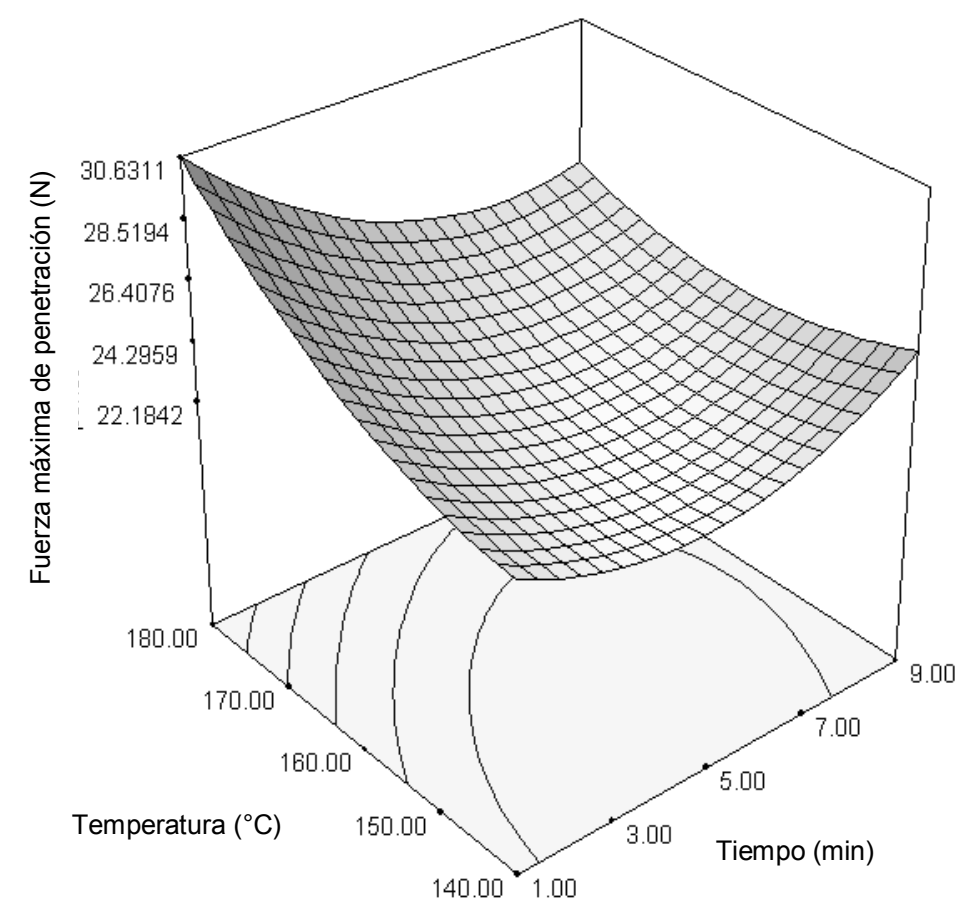

Fig. 2: Gráfico de superficie de respuesta para la Fuerza de Penetración

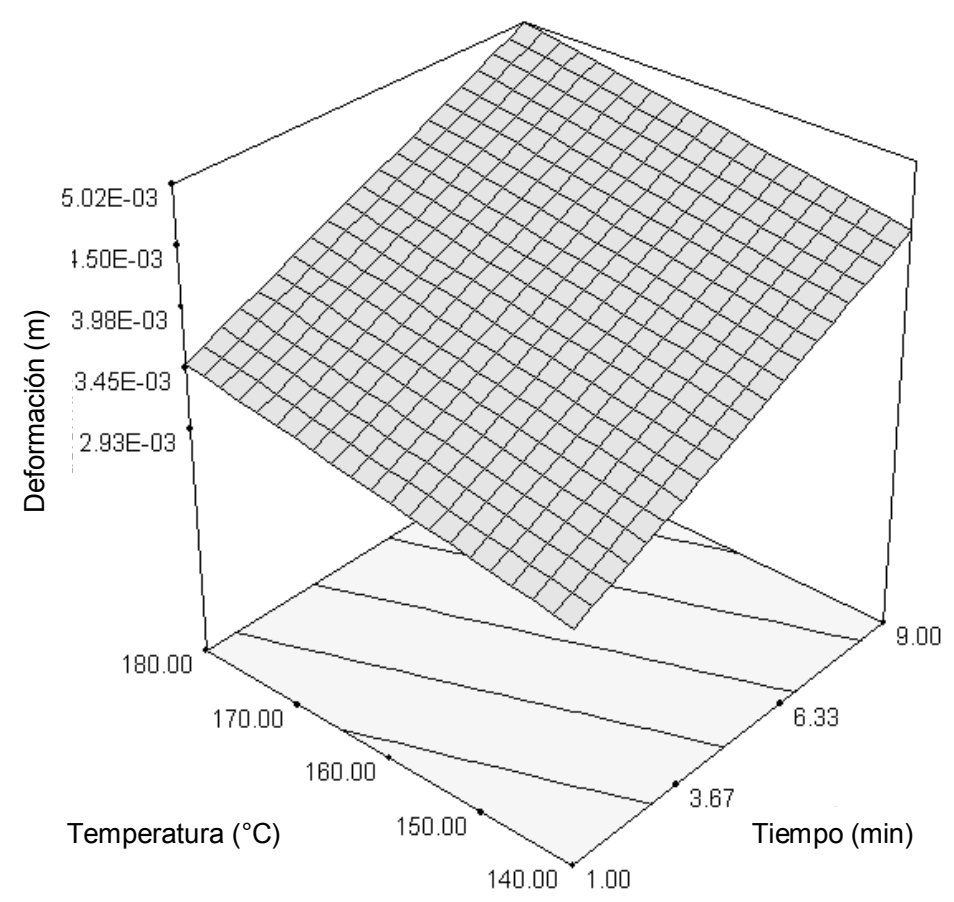

Fig. 3: Gráfico de superficie de respuesta para la deformación

Con los resultados obtenidos, utilizando el modelo de superficie de respuesta aplicado, se puede apreciar cómo el tiempo y la temperatura del proceso influyen significativamente en la deformación del producto cuando se somete a una prueba de penetración con un vástago cilíndrico y es evidente que a medida que ambos factores se incrementan, aumenta la deformación, debido a que el producto está casi seco y posee una costra crocante

Para el caso de la pendiente calculada en esta prueba, el modelo obtenido es el siguiente: 


$$
E_{a p} \equiv 663434-120667 t+276.67 T+67677 t^{2}-809.39 T^{2}-730.00 t T
$$

Mediante la superficie respuesta y aplicando el modelo anterior, puede observarse que a $t=1 \mathrm{~min}$. y $T=180^{\circ} \mathrm{C}$ se obtiene el valor más elevado de la pendiente ó módulo de elasticidad aparente, $E_{a p}$ mientras que en los extremos opuestos ( $t=9 \mathrm{~min}$. tanto a $T=140^{\circ} \mathrm{C}$ como $T=180^{\circ} \mathrm{C}$ ) se obtienen los valores más bajos. Un comportamiento similar (disminución de la "elasticidad"), fue reportado por Krokida et al. (2001) cuando estudiaron la influencia del proceso de freído sobre las propiedades viscoelásticas de papas a la francesa en el intervalo de temperatura de 150 a $190{ }^{\circ} \mathrm{C}$ y tiempos hasta $20 \mathrm{~min}$.

En la figura se aprecia además que a $t=9$ min, prácticamente no existe influencia de la temperatura.

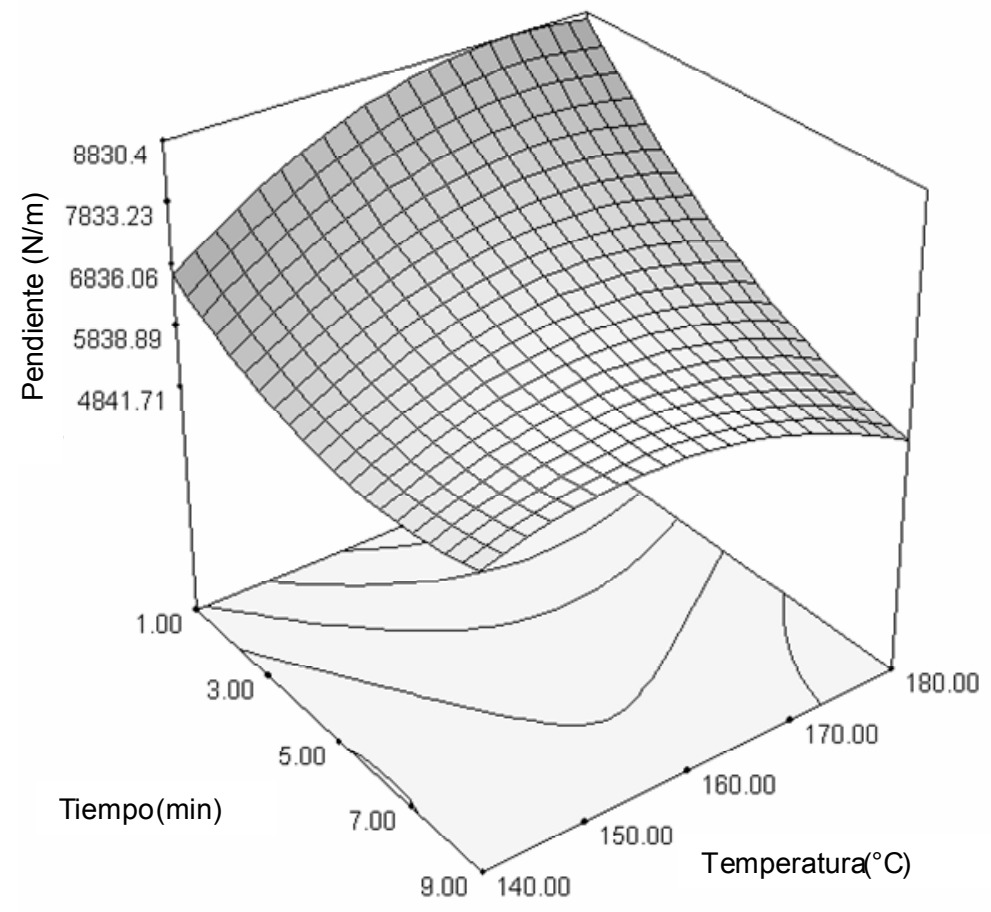

Fig. 4: Gráfico de superficie de respuesta para la pendiente

\section{CONCLUSION}

Los factores de proceso, tiempo de freído (intervalo de 1 a 9 min.) y temperatura del aceite (intervalo de 140 a $180^{\circ} \mathrm{C}$ ) afectan los parámetros texturales (fuerza de fractura, fuerza máxima de penetración, la deformación y la pendiente) durante el freído, en la primera etapa (1 a 4 min.) se presenta la gelatinización de los almidones en el trozo de ñame lo que produce ablandamiento de sus tejidos. Posteriormente, en la segunda etapa, se forma la costra (5 a 6 min.) y por último el endurecimiento a tiempos mayores de $7 \mathrm{~min}$. El tiempo de freído afecta la textura del producto, de forma tal que su incremento provoca un aumento en la fuerza necesaria para lograr su fractura, de acuerdo con el análisis de resultados, el tiempo y la temperatura de freído para obtener un producto con una textura ideal es de 5 minutos y $160^{\circ} \mathrm{C}$.

\section{REFERENCIAS}

Aguilera, J.M.; Fritura de Alimentos. Temas en Tecnología de Alimentos. Volumen I, Capítulo 5, Alfaomega, CYTED, p.p. 187-214 (1997). 
Anderson, A. y otros cuatros autores; Effect of preheating on potato texture. Critical Reviews in Food Science and Nutrition: 34(3), 229-251 (1994).

Blumenthal, M.M.; A new look at the chemistry and physics of deep-fat frying. Journal Food Technology: 45(2), 68-71 (1991).

Bradbury, H. y W.D. Holloway; Chemistry of tropical root croops: significance for nutrition and agriculture in the pacific, Monograph $\mathrm{N}^{\circ} 6$, Australian Centre for International Agricultural Research (ACIAR), 201p, Canberra, Australia (1987).

Costell, E., S.M. Fiszman, y L. Durán; Propiedades Físicas I: Reología de sólidos y textura. Temas en Tecnología de Alimentos. Volumen I, Capítulo 6, Alfaomega, CYTED, pp 215-260 (1997).

Cooursey, D.G. y P.H. Haynes; Root crops and their potential as in the tropics, World Crops: 22(4), 261-265 (1970).

FAO/FAOSTAT; Agriculture data, Food and agriculture organizations of the united Nations (2002), http//apps.fao.org/collections. Acceso: 17 de junio (2007).

Gacula, M.C. y J. Singh; Statistical methods in food and consumer research. New York, Academic Press, 214-272 (1984).

Ikoko, J. y V. Kuri; Osmotic pre-treatment effect on fat intake reduction and eating quality of deepfried plantain. Food Chemistry: 102(2), 523-531 (2007).

Krokida. M.K., V. Oreopoulou, Z.B. Maroulis y D. Marinos-Kouris; Viscoelastic behaviour of potato strips during deep fat frying. Journal of Food Engineering: 48(3), 213-218 (2001).

Minagricultura; Ministerio de Agricultura y Desarrollo Rural, Anuario estadístico del sector agropecuario. Bogotá (2003).

Montgomery, L.; Diseño y análisis de experimentos, México. Limusa-Wiley, $2^{\circ}$ edición, p.p. 225-270 (2002).

ONU; Raíces, tubérculos, plátanos y bananas en la nutrición humana, In Colección FAO: Alimentación y nutrición humana, N²4, 196p, Roma, Italia (1990).

Pedreschi, F. y P. Moyano; Oil uptake and texture development in fried potato slices. Journal of Food Engineering: 70(3), 557-563 (2005).

Rodríguez, G.; Breve reseña del ñame en Colombia. En: Corporación Colombiana de Investigación Agropecuaria (CORPOICA) (1996) http://agronomia.unal.edu.co/documentos/Volumen23-2.pdf. Acceso: 4 de septiembre (2007).

Sagui, I.S. y E.J. Pinthus; Oil uptake during deep-fat frying: Factors and mechanisms. Journal Food Technology: 49(4), 142-145 (1995).

Sagui, I.S., G. Ufheil y S. Livings; Oil uptake in deep-fat frying. Review, OCL, 5(1), 30-35. En: Transport Phenomena in Food Processing, J. Welti-Chanes (1998).

Taiwo, K.A. y O.D. Baik; Effects of pre-treatments on the shrinkage and textural properties of fried sweet potatoes. LWT- Food Science and Technology: 40(4), 661-668 (2007). 